\title{
Structural and functional changes of the coronary arteries in elderly senile patients with essential hypertension
}

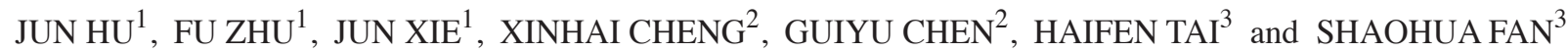 \\ ${ }^{1}$ Geriatric Department, Xuhui Central Hospital, Shanghai 200031; ${ }^{2}$ Medical Institute, Nantong University, Jiangsu 226019; \\ ${ }^{3}$ Medical Institute, Jiangsu University, Jiangsu 212013, P.R. China
}

Received March 28, 2013; Accepted July 29, 2013

DOI: $10.3892 / \mathrm{mmr} .2013 .1681$

\begin{abstract}
The aim of this study was to evaluate the effect of aging on the changes to the structure and function of coronary arteries in senile elderly patients with essential hypertension. Patients (aged 60-80 years) were divided into three groups. The 195 hypertensive patients were divided into four sub-groups according to the duration of hypertension. The changes to the coronary arteries (left and right) of all those patients were tested using the following index by 64 coronary computed tomography (CT) scans. The $24 \mathrm{~h}$ systolic blood pressure (SBP) and other blood biochemical parameters were assayed for all patients. We found that the value of the body mass index (BMI), total cholesterol (TC) and low density lipoproteins (LDL) were lower, but age and high density lipoproteins (HDL) were higher in the group of very elderly patients with hypertension (Group I; P<0.05) compared with those of a group of elderly patients with hypertension (Group III). The left anterior descending branch calcification score $\left(\mathrm{CS}_{\mathrm{LAD}}\right)$, total calcification score $\left(\mathrm{CS}_{\mathrm{T}}\right)$, pulse pressure (PP), the left main branch calcification score $\left(\mathrm{CS}_{\mathrm{LM}}\right)$, the left circumflex branch calcification score $\left(\mathrm{CS}_{\mathrm{LCX}}\right)$ were significantly increased in Group I compared with Group III $(\mathrm{P}<0.01$ and $\mathrm{P}<0.05$, respectively). In addition, the $24 \mathrm{~h}$ SBP value for Group I was higher than in the 'very elderly without hypertension' group (Group II). Hence, in elderly patients, a decrease in the levels of BMI, HDL, TC and LDL accompanies aging. Furthermore, the decline of arterial compliance and increase in arterial stiffness develops with age. Aging is more likely to lead to atherosclerosis in the coronary arteries, particularly in the left main coronary artery and its main branches. Aging is an uncontrollable risk factor, which plays a crucial role in coronary artery atherosclerosis.
\end{abstract}

Correspondence to: Dr Fu Zhu, Geriatric Department, Xuhui Central Hospital, No. 966 Huaihai Road, Xuhui, Shanghai 200031, P.R. China

E-mail: zhufu@medmail.com.cn

Key words: aging, CT scan of coronary artery, intima-media thickness, pulse pressure, hypertension

\section{Introduction}

Essential hypertension is a globally distributed disease and there are approximately one billion patients with essential hypertension in the world. Hypertension is a common chronic disease and it is the most important risk factor for the occurrence of cardiovascular disease. Furthermore, hypertension may lead to stroke, myocardial infarction, heart failure and chronic kidney disease, as well as other serious complications (1-3). As China has gradually developed an aging society, the process of aging has been accelerating. Amongst the risk factors found in elderly patients with cardiovascular diseases, aging is one of the main features which is different to the non-elderly factors (1-5). Morbidity due to hypertension in elderly patients, particularly at an advanced age, was $>80 \%(6,7)$. In this study, we aimed to, via the coronary arteries and its main branches, evaluate the structure and function and understand the changes to the large arteries of elderly patients due to aging and the complications arising from hypertension.

\section{Materials and methods}

Subjects and grouping. In total, 238 inpatients were enrolled in this study from March 2010 to March 2012. The diagnosis of hypertension conformed to the ' 2005 Guidelines for Prevention and Treatment of Hypertension in China (rev.)'. All patients with diabetes mellitus, persistent atrial fibrillation, hypertrophic cardiomyopathy, dilated cardiomyopathy, heart failure, serious infections and severe liver and kidney injuries within the last month were excluded from this study. The study was approved by the Ethics Committee of Xuhui Central Hospital (Shanghai, China) and written informed consent was obtained from the patients.

Patients $>80$ years old were considered to be 'very elderly', and those $>60$ years old but $<80$ years old were considered to be 'elderly'. Furthermore, in line with whether there was the complication of hypertension, the patients were divided into the 'very elderly with hypertension' group (Group I; 119 patients, consisting of 64 males and 55 females), the 'very elderly group without hypertension' group (Group II), 43 patients, consisting of 24 males and 19 females), and the 'elderly patients with hypertension' group (Group III, 76 patients, consisting of 45 males and 31 females). All 195 patients with hypertension were divided into four sub-groups according to their history 
Table I. Basic situation, metabolism and blood pressure in the three groups.

\begin{tabular}{|c|c|c|c|c|c|}
\hline Variable & Group I & Group II & Group III & $\begin{array}{c}\text { P-value } \\
\text { (Group I vs. Group II) }\end{array}$ & $\begin{array}{c}\text { P-value } \\
\text { (Group I vs. Group III) }\end{array}$ \\
\hline (Male/female) & $119(64 / 55)$ & $43(24 / 19)$ & $76(45 / 31)$ & $>0.05$ & $>0.05$ \\
\hline Age (years) & $84.72 \pm 3.12$ & $85.32 \pm 4.12$ & $72.59 \pm 2.48$ & $>0.05$ & $>0.05$ \\
\hline Smoking (\%) & $17 / 119$ & $7 / 43$ & $14 / 76$ & 0.720 & 0.505 \\
\hline BMI $\left(\mathrm{kg} / \mathrm{m}^{2}\right)$ & $23.70 \pm 0.49$ & $23.77 \pm 1.61$ & $24.59 \pm 0.42$ & 0.749 & 0.002 \\
\hline TG (mmol/l) & $1.33 \pm 0.09$ & $1.10 \pm 0.09$ & $1.61 \pm 0.14$ & 0.283 & 0.098 \\
\hline $\mathrm{TC}(\mathrm{mmol} / \mathrm{l})$ & $4.31 \pm 0.09$ & $4.07 \pm 0.22$ & $4.70 \pm 0.14$ & 0.203 & 0.007 \\
\hline $\mathrm{HDL}(\mathrm{mmol} / \mathrm{l})$ & $1.35 \pm 0.04$ & $1.38 \pm 0.10$ & $1.19 \pm 0.03$ & 0.798 & 0.017 \\
\hline $\mathrm{LDL}(\mathrm{mmol} / \mathrm{l})$ & $2.28 \pm 0.08$ & $2.20 \pm 0.14$ & $2.79 \pm 0.11$ & 0.569 & 0.001 \\
\hline FPG (mmol/l) & $4.86 \pm 0.08$ & $5.15 \pm 0.33$ & $5.22 \pm 0.24$ & 0.805 & 0.151 \\
\hline HbA1c $(\%)$ & $5.86 \pm 0.06$ & $5.88 \pm 0.11$ & $6.00 \pm 0.18$ & 0.935 & 0.413 \\
\hline 24 h SBP (mmHg) & $129.02 \pm 1.74$ & $118.39 \pm 3.00$ & $125.77 \pm 1.96$ & 0.009 & 0.298 \\
\hline $24 \mathrm{~h} \mathrm{DBP}(\mathrm{mmHg})$ & $72.31 \pm 1.06$ & $62.51 \pm 1.40$ & $71.54 \pm 1.26$ & $<0.000$ & 0.784 \\
\hline
\end{tabular}

The values are provided as the means \pm SE. Group I, very elderly patients with hypertension; Group II, very elderly patients without hypertension; Group III, elderly patients with hypertension. BMI, body mass index; TG, triglyceride; TC, total cholesterol; HDL, high density lipoprotein; LDL, low density lipoprotein; FPG, fasting blood-glucose; HbA1c, glycosylated hemoglobin A1c; 24 h SBP, 24 h systolic blood pressure; $24 \mathrm{~h}$ DBP, $24 \mathrm{~h}$ diastolic blood pressure.

Table II. Comparison of carotid artery vascular structure and PP in the three groups.

\begin{tabular}{|c|c|c|c|c|c|}
\hline Variable & $\begin{array}{l}\text { Group I } \\
(\mathrm{n}=119)\end{array}$ & $\begin{array}{l}\text { Group II } \\
(\mathrm{n}=43)\end{array}$ & $\begin{array}{l}\text { Group III } \\
(\mathrm{n}=76)\end{array}$ & $\begin{array}{c}\text { P-value } \\
\text { (Group I vs. Group II) }\end{array}$ & $\begin{array}{c}\text { P-value } \\
\text { (Group I vs. Group III) }\end{array}$ \\
\hline IMT (mm) & $0.84 \pm 0.10$ & $0.84 \pm 0.19$ & $0.80 \pm 0.13$ & 0.062 & 0.111 \\
\hline PP (mmHg) & $56.89 \pm 1.24$ & $56.30 \pm 2.90$ & $51.32 \pm 1.13$ & 0.693 & 0.041 \\
\hline
\end{tabular}

The values are provided as the means \pm SE. Group I, very elderly patients with hypertension; Group II, very elderly patients without hypertension, Group III, elderly patients with hypertension. IMT, carotid artery intima-media thickness; PP, pulse pressure.

of hypertension: Group A: $<10$ years (44 patients); Group B: $>10$ but $<20$ years (48 patients); Group C: $>20$ but $<30$ years (60 patients); Group D: $>30$ but $<40$ years (43 patients).

General information. We recorded the patients' age, gender, height $(\mathrm{H}, \mathrm{cm})$, weight $(\mathrm{W}, \mathrm{kg})$, history and course of hypertension, history of smoking, fasting blood-glucose (FBG), glycosylated hemoglobin A1c (HbA1c), total cholesterol (TC), triglyceride (TG), low density lipoproteins (LDL) and high-density lipoproteins (HDL). The equation used to calculate the body mass index was: $\mathrm{BMI}=10,000 \mathrm{x} \mathrm{W} / \mathrm{H}^{2}$.

Determination of carotid artery intima-media thickness (IMT). The color Doppler ultrasound imaging system, and 10.0 MHz linear array probe (Siemens, Washington, DC, USA) were used. The patient was placed into a supine position, and the probe was placed under the posterior angulus frontalis of the neck. The common carotid artery was probed vertically. At the end of the left ventricular diastolic period (when the synchronous ECG showed R waves), the images, $10 \mathrm{~cm}$ below the carotid sinus, were fixed. Three cardiac cycles were measured on each side. The posterior wall of the common carotid artery revealed two parallel bright lines separated by a relatively low echo. There was an average of six measurements of vertical distances among them, calculated at the left and right sides of the carotid artery, for the IMT.

Determination of the coronary artery calcification score (CS). The Philips Brilliance spiral computed tomography (CT) scanner (Philips Healthcare, Best, The Netherlands) was adopted, and the technique of ECG anterior gating was used to scan the heart (8). The thickness of the scanning layer was $2 \mathrm{~mm}(120 \mathrm{kV}, 300 \mathrm{~mA})$ with a scanning speed of $0.5 \mathrm{sec}$. A Philips EBW workstation was adopted to analyze the calcification of the coronary arteries. The coronary arteries were divided into four sections; the left main coronary artery (LM), the left anterior descending coronary artery (LAD), the left circumflex coronary artery (LCX) and the right coronary artery (RCA), to analyze the degree of coronary artery calcification. The degree of coronary artery calcification was judged, and the determination of CS was performed using methods such as Agatston scoring (4,5). A coronary CT value $>130$ HU was considered to be the calcification standard. The CS for each branch of the coronary arteries was automatically 
Table III. Calcification scores for the three groups.

\begin{tabular}{lccccc}
\hline CS & $\begin{array}{c}\text { Group I } \\
(\mathrm{n}=119)\end{array}$ & $\begin{array}{c}\text { Group II } \\
(\mathrm{n}=43)\end{array}$ & $\begin{array}{c}\text { Group III } \\
(\mathrm{n}=76)\end{array}$ & $\begin{array}{c}\text { P-value } \\
\text { (Group I vs. Group II) }\end{array}$ & $\begin{array}{c}\text { P-value } \\
\text { (Group I vs. Group III) }\end{array}$ \\
\hline RCA & $149.80 \pm 56.44$ & $130.06 \pm 64.70$ & $59.54 \pm 23.94$ & 0.992 & 0.127 \\
LM & $46.71 \pm 15.12$ & $48.40 \pm 26.82$ & $13.80 \pm 9.13$ & 0.949 & 0.041 \\
LAD & $173.29 \pm 23.37$ & $254.27 \pm 67.39$ & $102.12 \pm 31.90$ & 0.258 & 0.005 \\
LCX & $59.06 \pm 14.88$ & $133.07 \pm 71.39$ & $70.77 \pm 42.78$ & 0.605 & 0.033 \\
T & $431.73 \pm 83.48$ & $578.18 \pm 139.12$ & $244.77 \pm 85.93$ & 0.158 & 0.002 \\
\hline
\end{tabular}

The values are provided as the means \pm SE. Group I, very elderly patients with hypertension; Group II, very elderly patients without hypertension; Group III, elderly patients with hypertension. CS, calcification score; RCA, right coronary artery; LM, left main branch; LAD, left anterior descending branch; LCX, left circumflex branch; T, total.

calculated using a computer. Total calcification scores $\left(\mathrm{CS}_{\mathrm{T}}\right)$ for each branch were also determined.

Determination of pulse pressure $(P P), 24$ h average systolic blood pressure (24 h SBP) and $24 \mathrm{~h}$ average diastolic blood pressure $(24 h \mathrm{DBP})$. An ambulatory blood pressure monitor (SunTech Medical, Morrisville, NC, USA) was adopted to monitor the ambulatory blood pressure, every 20 min during the daytime, and every 30 min during the night. The equation used to calculate the PP was: SBP-DBP $=$ PP. The $24 \mathrm{~h}$ SBP and $24 \mathrm{~h}$ DBP were also recorded.

Statistical analysis. The database was established, and the statistical descriptions are provided as the means $\pm \mathrm{SE}$. Hypothesis testing was performed using the Chi-square test, the rank-sum test, analysis of variance (ANOVA) and bivariate correlation analysis. The statistical software used in this study was SPSS 15.0 (SPSS, Inc., Chicago, IL, USA). P<0.05 was considered to indicate a statistically significant difference.

\section{Results}

Comparison of basic conditions, metabolism and BP. There were no statistical differences between the smoking status, TG, FPG, HbA1c, $24 \mathrm{~h}$ SBP and 24 h DBP (P>0.05), while there were marked differences in age, BMI, TC and LDL between Groups I and III $(\mathrm{P}<0.01)$, while there was a statistically significant difference in HDL between the two groups $(\mathrm{P}<0.05$; Table I). There were statistical differences in $24 \mathrm{~h} \mathrm{SBP}$ and $24 \mathrm{~h}$ DBP between Groups I and II $(\mathrm{P}<0.01)$, while there were no statistical differences in age, smoking status, BMI, TG, TC, HDL, LDL, FPG and HbA1 between the two groups $(\mathrm{P}>0.05$; Table I).

Comparison of carotid artery structure and PP. There were no significant statistical differences in IMT and PP between Groups I and II (P>0.05; Table II). There was a statistical difference in PP between Groups I and III $(\mathrm{P}<0.05)$, while there were no statistical differences in IMT between the two groups $(\mathrm{P}>0.05$; Table II).

Comparison of CS. There were no statistical differences in $\mathrm{CS}_{\mathrm{RCA}}, \mathrm{CS}_{\mathrm{LM}}, \mathrm{CS}_{\mathrm{LAD}}, \mathrm{CS}_{\mathrm{LCX}}$ and $\mathrm{CS}_{\mathrm{T}}$ between Groups I and II
Table IV. The relevance between the course of hypertension and blood pressure, CS and PP.

\begin{tabular}{lcc}
\hline Variables & $\mathrm{r}$ & P-value \\
\hline $\mathrm{CS}_{\mathrm{RCA}}$ & 0.149 & 0.051 \\
$\mathrm{CS}_{\mathrm{LM}}$ & 0.142 & 0.066 \\
$\mathrm{CS}_{\mathrm{LAD}}$ & 0.110 & 0.151 \\
$\mathrm{CS}_{\mathrm{LCX}}$ & 0.133 & 0.083 \\
$\mathrm{CS}_{\mathrm{T}}$ & 0.160 & 0.036 \\
$24 \mathrm{~h} \mathrm{SBP}$ & 0.223 & 0.003 \\
$\mathrm{PP}$ & 0.138 & 0.072
\end{tabular}

$\mathrm{CS}_{\mathrm{RCA}}$, calcification score right coronary artery; $\mathrm{CS}_{\mathrm{LM}}$, calcification score left main branch; $\mathrm{CS}_{\mathrm{LAD}}$, calcification score left anterior descending branch; $\mathrm{CS}_{\mathrm{LCX}}$, calcification score left circumflex branch; $\mathrm{CS}_{\mathrm{T}}$, total calcification score; $24 \mathrm{~h} \mathrm{SBP}, 24 \mathrm{~h}$ systolic blood pressure; $\mathrm{PP}$, pulse pressure.

( $\mathrm{P}>0.05$; Table III). There were significant statistical differences in $\mathrm{CS}_{\mathrm{LAD}}$ and $\mathrm{CS}_{\mathrm{T}}$ in Groups I and III $(\mathrm{P}<0.01)$, and there were statistical differences in $\mathrm{CS}_{\mathrm{LM}}$ and $\mathrm{CS}_{\mathrm{LCX}}(\mathrm{P}<0.05)$, while there were no statistically significant differences found for $\mathrm{CS}_{\mathrm{RCA}}(\mathrm{P}>0.05$; Table III).

Effects of the course of hypertension on BP, CS and PP. Bivariate correlation analysis revealed that there was positive correlation between $\mathrm{CS}_{\mathrm{T}}$ and the course of hypertension $(\mathrm{r}=0.160, \mathrm{P}<0.05)$. Furthermore, there was positive correlation between $24 \mathrm{~h} \mathrm{SBP}$ and the course of hypertension $(r=0.223$, $\mathrm{P}<0.01)$. However, there was no significant correlation between PP and the course of hypertension $(\mathrm{r}=0.138, \mathrm{P}>0.05)$ (Table IV).

\section{Discussion}

In this study, we observed indicators of atherosclerosis in the coronary arteries and the carotid artery, and explored the effect of cardiovascular risk factors, such as aging, blood pressure and lipid metabolism, on the structure and function of the large arteries in 'very elderly' patients with hypertension. 


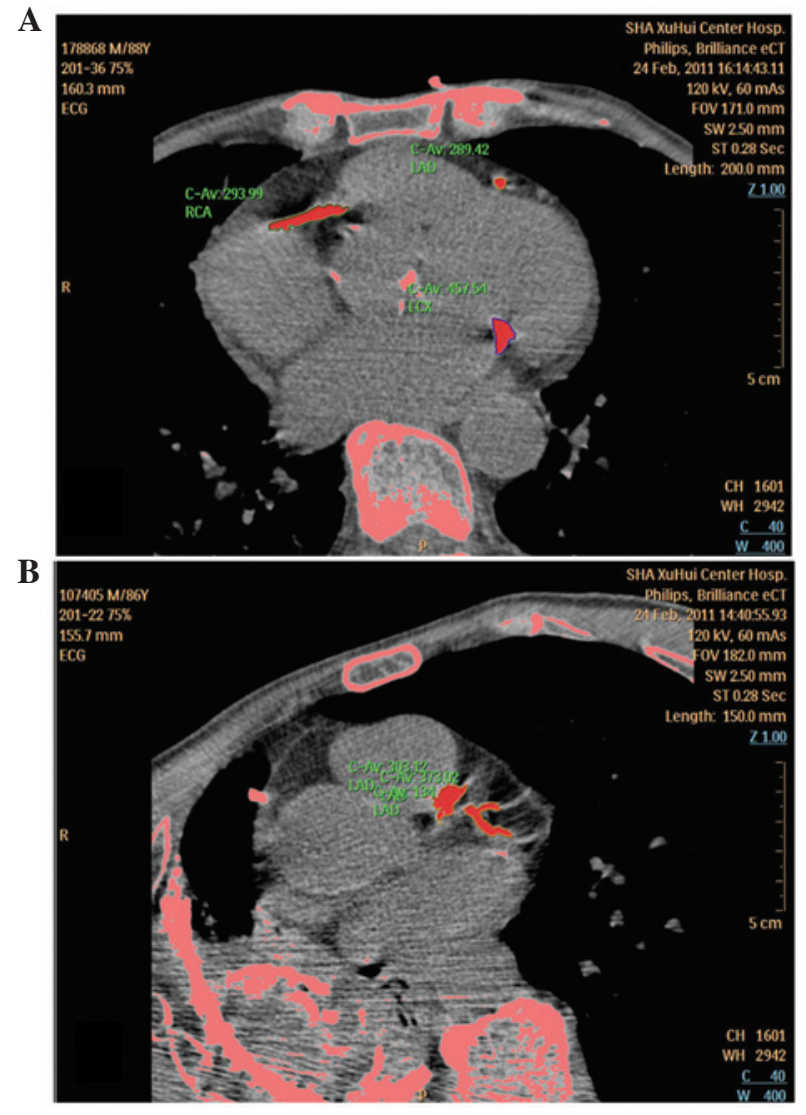

Figure 1. Results of coronary artery calcification score detected by spiral CT scanner. Coronary artery was divided into four sections; the left main coronary artery, the left anterior descending coronary artery, the left circumflex coronary artery and the right coronary artery, in order to analyze the calcification of the coronary artery. (A) Very elderly patient with hypertension (Group I); (B) very elderly patients without hypertension (Group II). CT, computed tomography.

Aging is an independent risk factor for cardiovascular diseases. The incidence of hypertension increases with age. In this study, we included 162 'very elderly' patients aged $>80$ years. Amongst them, were 119 patients with hypertension, which was consistent with literature reports that the morbidity of hypertension in 'very elderly' patients is $>80 \%$. In this study, lipid metabolism, glucose metabolism and BMI levels were shown to be equivalent in 'very elderly' patients with or without hypertension. Epidemiological and clinical studies on BP and PP have demonstrated that the long-term exposure of the arterial vascular wall to hypertension leads to high arterial pressure. Under this state, vascular endothelial function changed secondarily, the synthesis and release of NO was decreased, endothelium-dependent vasodilation was weakened, hence an increase in BP and PP. All hypertension patients were admitted into Xuhui Central Hospital and administered a regular antihypertensive therapy, with a good compliance. In Groups I and II, $24 \mathrm{~h}$ SBP were 129.02 \pm 1.74 and $118.39 \pm 3.00 \mathrm{mmHg}$, respectively. The BP in Group I was controlled more ideally. This may be due to the fact that there was no difference in PP between Groups I and II. In this study, PP may reflect the arterial function.

In this study, there were no differences between Groups I and II. Nonetheless, there were differences between Groups I and III, and the degree of calcification in the LAD was
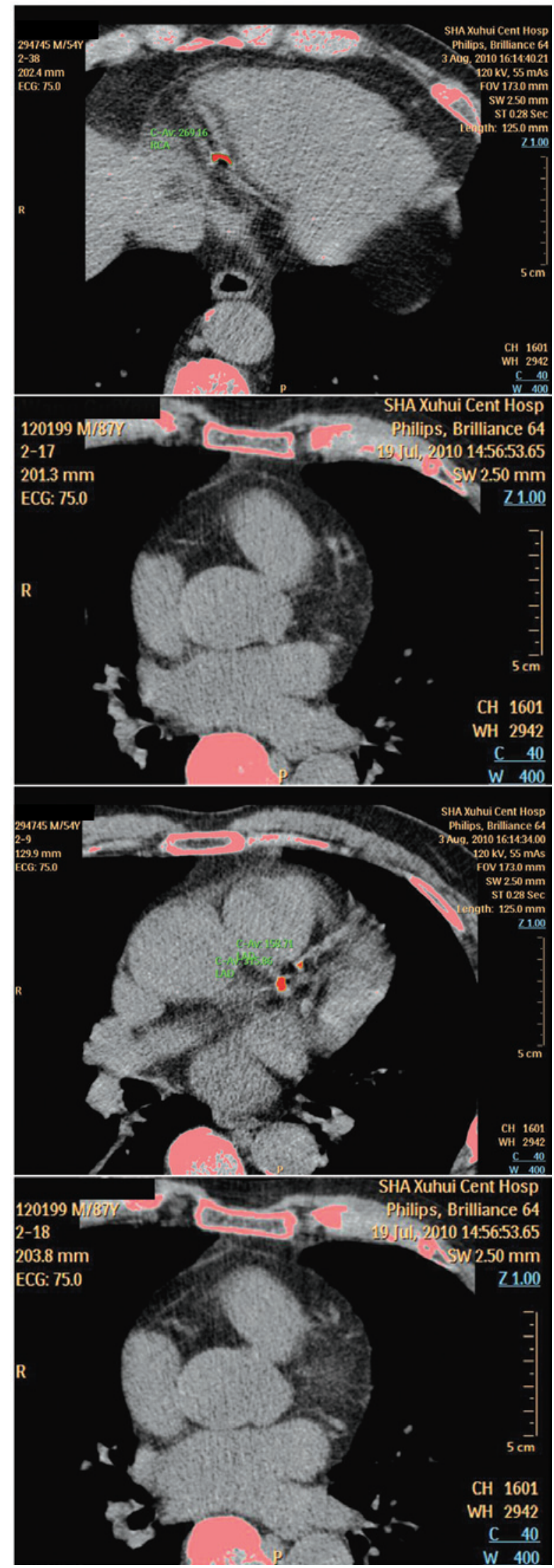

Figure 2. Results of the coronary artery calcification score for a group of elderly patients with hypertension (group III) detected by spiral CT scanner. The coronary artery was divided into four sections; namely the LM coronary artery, the LAD coronary artery, the LCX coronary artery and the RCA, to analyze the calcification of the coronary artery. LM, LAD, LCX and RCA did not contain significant levels of calcified plaque. CT, computed tomography; LM, left main; LAD, left anterior descending; LCX, left circumflex; RCA, right coronary artery. 
particularly serious. Meanwhile, the degree of calcification in the LCX and LM also increased with age. This indicated that the coronary artery calcification in elderly patients caused by aging was clear and should not be ignored. Many studies have verified, from the pathogenesis and histopathology of atherosclerosis, that the arterial calcification was closely related to arteriosclerosis, and CS was relevant to the possibility of coronary artery stenosis and increased numbers of stenotic branches. The higher the CS, the higher the incidence of coronary artery stenosis, and the greater the numbers of vascular branches involved. Other studies have considered that CS may predict the risks of myocardial infarction and cardiovascular mortalities $(9,10)$. Thus, coronary artery lesions incurred by aging or risks produced by succedent cardiovascular events may not be less than those caused by hypertension. It had been known that age, gender, smoking, hypertension, hyperlipemia, hyperuricemia and diabetes mellitus were relevant factors for coronary artery disease (9). Among them, gender and age are unchangeable, while other risk factors may be minimized. The subjects of this study were all in-hospital patients. The treatments, such as regulating lipids, controlling BP, regulating serum uric acid and improving insulin resistance may cause biases relevant to blood fat, BP, blood sugar and coronary arteriosclerosis, which was a limitation of this study.

Atherosclerosis is a disease with multiple risk factors. Amongst them, age is an unchangeable risk factor. As one of the independent risk factors of cardiovascular diseases, the age-related changes should be targets for treatment and intervention for elderly patients with cardiovascular diseases in the future.

\section{Acknowledgements}

This study was supported by a grant to $\mathrm{Fu} \mathrm{Zhu}$ from the Supported by Science and Technology Commission of Shanghai Municipality (no. 10411968700).

\section{References}

1. Yang L, Zou J, Zhang M, et al: The relationship between thyroid stimulating hormone within the reference range and coronary artery disease: impact of age. Endocr J March 2013 (Epub ahead of print).

2. Maraj I, Makaryus JN, Ashkar A, McFarlane SI and Makaryus AN: Hypertension management in the high cardiovascular risk population. Int J Hypertens 2013: 382802, 2013.

3. Repossini A, Tespili M, Saino A, et al: Hybrid revascularization in multivessel coronary artery disease. Eur J Cardiothorac Surg Feb 2013 (Epub ahead of print).

4. Bild DE, Detrano R, Peterson D, et al: Ethnic differences in coronary calcification: the Multi-Ethnic Study of Atherosclerosis (MESA). Circulation 111: 1313-1320, 2005.

5. Manolio TA, Arnold AM, Post W, et al: Ethnic differences in the relationship of carotid atherosclerosis to coronary calcification: the Multi-Ethnic Study of Atherosclerosis. Atherosclerosis 197: 132-138, 2008.

6. Fu S, Liu Y, Zhu B, et al: Prognostic abilities of different calculation formulas for the glomerular filtration rate in elderly Chinese patients with coronary artery disease. Clin Interv Aging 8: 229-237, 2013.

7. Palma-Reis I, Vais A, Nelson-Piercy C and Banerjee A: Renal disease and hypertension in pregnancy. Clin Med 13: 57-62, 2013.

8. Faria Alves M, Ferreira AM, Cardoso G, Saraiva Lopes R, Correia MD and Machado Gil V: Pre- and post-test probability of obstructive coronary artery disease in two diagnostic strategies: Relative contributions of exercise ECG and coronary CT angiography. Rev Port Cardiol 32: 211-218, 2013 (In English and Portuguese).

9. Bellasi A, Lacey C, Taylor AJ, et al: Comparison of prognostic usefulness of coronary artery calcium in men versus women (results from a meta- and pooled analysis estimating all-cause mortality and coronary heart disease death or myocardial infarction). Am J Cardiol 100: 409-414, 2007.

10. Chen Q, Liu Y, Yin Y, Huang W, Li G and Ke D: Relationship between metabolic syndrome (MS) and coronary heart disease (CHD) in an aged group. Arch Gerontol Geriatr 46: 107-115, 2008. 